\title{
On Number of k-Element in Open and Clopen Topological Space With Corresponding Graph for $n \leq 4$
}

\author{
Francis Moses Obinna \\ Department of Mathematics, Federal University of Technology, Minna, Nigeria
}

Adeniji A. O.

Department of Mathematics, University of Abuja, Abuja, Nigeria

\begin{abstract}
In this paper, we determine the number of elements defined on each topology of a topological space for open and clopen topological spaces $T(n)$ for $1 \leq n \leq 4$, which is called k-element. A graph of the relationship between topologies for open and clopen set for $n \leq 3$ are discussed. Hence; $T(n, k)$ is clopen for $n \geq 1$ if and only if $k=2^{n}$.

Keywords: K-element; Open topological space; Clopen topological space.

\section{(c) (1) CC BY: Creative Commons Attribution License 4.0}

\section{Introduction}

Listing [1], introduced the term topology in 1847, although it was not until 1848 that the idea of topology space was developed. Sharp [2], and Kolli [3], establish the number of topology on a finite set in 1968, 2007 respectively. Stanley [4], studied the number of open sets of finite topologies in 1971. In 2006, Benoumhani [5] Compute T(n, k) for $2 \leq \mathrm{k} \leq 12$, and other results concerning $\mathrm{T}_{0}$ topologies on $\mathrm{X}$ for some open sets. In 2010, Benoumhani and Kolli [6] Compute $\mathrm{T}(\mathrm{n}, \mathrm{k})$ for $\mathrm{k} \leq 17$ and also the number of all unlabeled non- $\mathrm{T}_{0}$ topologies on $\mathrm{X}$ with $\mathrm{k}$ open sets, for 3 $\leq \mathrm{k} \leq 8$. Ali-Ahmad [7], enumerated the number of fuzzy clopen sets in arbitrary fuzzy topological space and also prove the number of all crisp fuzzy clopen sets in an arbitrary fuzzy topology in 2017. The online encyclopaedia of Sloane [8] gives the value of $\mathrm{T}(\mathrm{n})$ for $1 \leq n \leq 14$. The purpose of this paper is to enumerate the number of $\mathrm{k}$ elements in an open and clopen topological space for small value of $\mathrm{n}$. And also, to discuss the graphical relationship between topologies for open and clopen set for $\mathrm{n} \leq 3$.
\end{abstract}

\section{Preliminaries}

Throughout this paper $(X, \tau)$ means topological spaces on which no separation are assumed unless otherwise stated. Let $\mathrm{T}(\mathrm{n})$ denote the number of topologies on $X$ and $T(n, k)$ be the number of all topologies having kelements that can be defined topologies on $\mathrm{X}$. Let $\mathrm{A}$ be a subset of $(X, \tau)$ and $\tau^{c o}$ denote clopen topologies

\subsection{Definition}

A subset A of a topological space $(x, \tau)$ is said to be

(i) Open if $A \epsilon \tau$

(ii) Closed if $A^{c} \in \tau$

(iii) Clopen if $A$ is both open and closed i.e. $A \epsilon \tau$ and $A^{c} \in \tau$

(iv) Discrete if and only if $\mathrm{X}$ is the collection of subsets $\tau=P(X)$, where $\mathrm{P}(\mathrm{X})$ is the power set and $\mathrm{X}$ is any set.

(v) Trivial space if and only if the subset of the topology are the empty set and the entire space. i.e. $X$ and $\phi$.

(vi) Sierpinki if it has two points, with only one of it closed

\section{Number of k-Elements in an Open Topological Space for $\boldsymbol{n} \leq \mathbf{4}$}

In this section we obtain and illustrate our results with figures and table of values for k-elements on open topological spaces. In arrived at the k-elements on topological spaces, the power sets for $n=1,2,3$ and 4 are given as $2^{n}$ which are as follows; $2,4,8,16, \ldots$ respectively. The stoppage value for $k$ is dependent on the power set for each $n$, and the sum of all $T(n, k)$ gives the number of topologies on that set.

\subsection{Definition}

A topology on a set $X$ is a collection $\tau$ of subsets of $x$ which satisfy the following properties:

(i) The basis set $X$, and the empty set $\emptyset$ belongs to $\tau$

(ii) The union of any finite number of sets in $\tau$ belongs to $\tau$

(iii) The intersection of any two set in $\tau$ belongs to $\tau$

The pair $(X, \tau)$ is called topological space. 


\subsection{Example}

(i) For $n=1$

Let $X=\{a\}$

$$
\tau=\{\varnothing, X\}
$$

Equation (13) is open by definition

$$
\tau^{c}=\{X, \varnothing\}
$$

Equation (14) is open by definition

By definition $\{x, \emptyset\}$ are clopen since they are both open and closed in $(x, \tau)$

(ii) For $n=2$

Let $X=\{a, b\}$

$$
\begin{aligned}
\tau_{1} & =\{\varnothing, a, X\} \\
\tau_{2} & =\{\varnothing, b, X\} \\
\tau_{3} & =\{\varnothing, a, b, X\} \\
\tau_{4} & =\{\varnothing, X\}
\end{aligned}
$$

(iii) Let $X=\{a, b, c\}$ then

the number of topologies define on $X$ are 29

(iv) Let $X=\{a, b, b, d\}$ then

The numbers of topologies form on $\mathrm{X}$ are 335 .

Equation (3) is open by definition and the topologies formed on $\mathrm{X}$ are represented with Figure 3.3 the set has three forms of topologies which are $\tau_{1}$ and $\tau_{2}$ known as one non-trivial open topological space, they has three elements each. Therefore, $\mathrm{k}$ at this point has three elements. The next is $\tau_{3}$ it is known as discrete topology because it contained every element that can be form on $\mathrm{X}$. and it contains four elements $(k=4)$. And also, $\tau_{4}$ is known as the trivial topology. It is contained in every topological space formed on X. It contained two elements that is ( $k=$ 2).

Figure-3.3. Schematic nature of open topological spaces with two elements

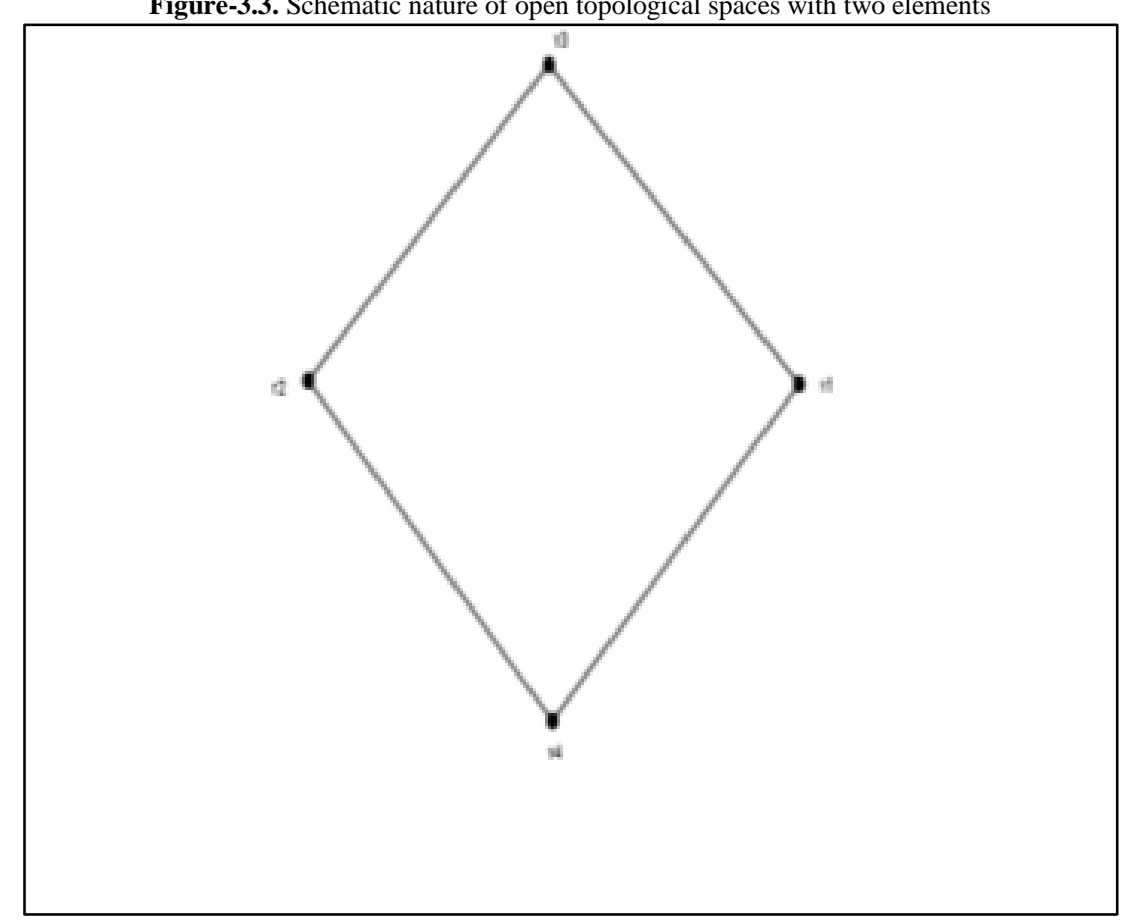

\subsection{Proposition}

Every subset $A \epsilon \tau$ is an open set in the topological space.

Proof follows from the definition.

\subsection{Theorem}

Given a set $\mathrm{X}$ with three elements $\{a, b\}$. Such that all topologies formed on $\mathrm{X}$ are open on $\mathrm{X}$, and satisfies the properties of a topological space. Then there exist three topological classes on X.

\subsection{Theorem}

An empty set of a topological space $(x, \tau)$ has only empty open set.

\subsubsection{Proof}

Given $(X, \tau)$, let $A \in X$ be an open set, $A \epsilon \tau$

$\emptyset \in A \in \tau$, Since $\emptyset$ is only set on $A, \quad \emptyset \in \tau$ 


\subsection{Remark}

For an empty set the numbers of topologies define on it is much unique. The only open set is the empty set itself. Therefore, it has a zero k-element. For a set with 2 elements, there are four distinct topologies on $X$, which one is discrete, one is trivial and the other two are sierpinski topologies. For set with 3 elements there are 29 distinct topologies on $X$, with only 9 non-equivalent topologies, in which one is discrete, one trivial. Similarly, for a set with 4 elements, there are 355 distinct topologies on $X$ with 33 non-equivalent topologies.

\subsection{Example}

Table 3.10: can be illustrated with the formula

$T(n)=\sum_{k=1}^{2^{n}} T(n, k)$ for $1 \leq k \leq 2^{n}$

If $n=1, X=\{a\}$. And the maximum value of $k=2^{n}$, which is $k=2^{1}=2$.

Therefore, the maximum values of $T(n, k)=T(1,2)$ we obtain our result as follow:

(4)

$$
T(1,1)=0, T(1,2)=1
$$

The sum of (4) gives

$$
T(1,1)+T(1,2)=0+1=1
$$

For $n=2$

$X=\{a, b\}$ the maximum value of $k=2^{2}=4$. Therefore, we have $T(2,4)$ as the stoppage value of $\mathrm{k}$

$T(2,1)=0, T(2,2)=1, T(2,3)=2, T(2,4)=1$

The sum of $(6)$ gives

$T(2,1)+T(2,2)+T(2,3)+T(2,4)$

$=0+1+2+1=4$

Similarly for $n=3$

$X=\{a, b, c\}$ and maximum value of $k=2^{3}=8$. Therefore, we have three as the set value and eight as the stoppage value of the topological space, that is $T(3,8)$ which is the last topological space with three elements, we analysis the result as follows:

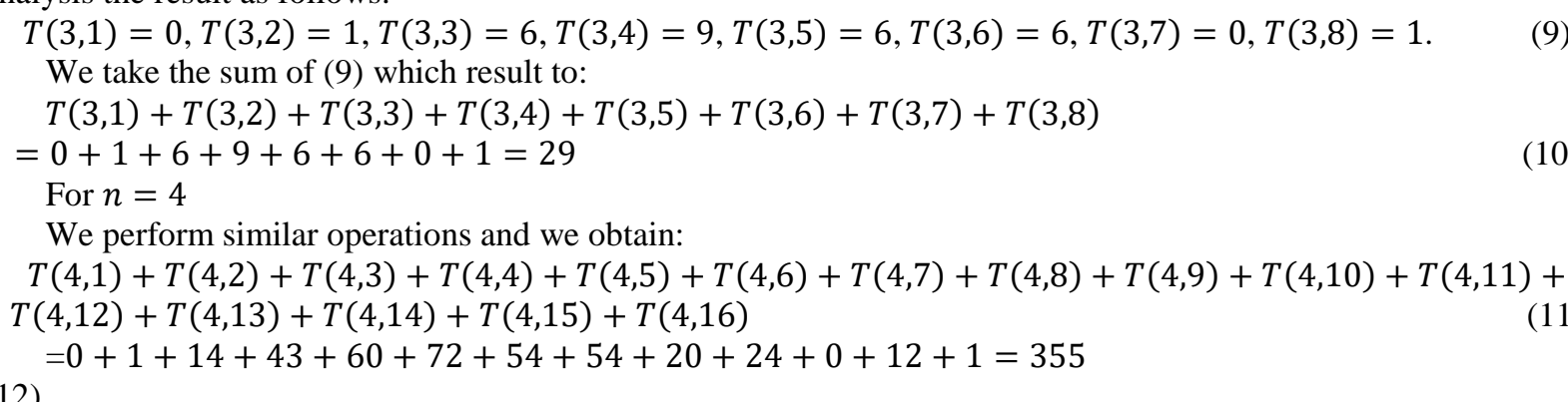

Hence, we then generalize that; for any given $T(n)$ where $k$ ranges from $1 \leq k \leq 2^{n}$

$T(n)=\left(T(n, 1)+T(n, 2)+T(n, 3)+\cdots \cdot+T\left(n, 2^{n}\right)\right)$

$T(n)=(T(n, 1)+T(n, 2)+T(n, 3)+\cdots .+T(n, k))$

So that $T(n)=\sum_{k=1}^{2^{n}} T(n, k)$.

\subsection{Theorem}

Given a set $\mathrm{X}$ with three elements $\{a, b, c\}$. Such that all topologies formed on $\mathrm{X}$ are open on $\mathrm{X}$, and satisfies the properties of a topological space. Then there exist nine topological classes on X.

\subsubsection{Proof}

Figure 3.10 is a graph of the relation between open topological spaces with three elements. The graph shows how topological spaces of set with three elements are linked to the other. $\tau 1$ is distinct; it contains all the subset formed in every other topology. While $\tau 27$ is a trivial topological space, every other topology contains it. The topologies $\tau 24-\tau 29$ are classes of topologies with four non-trivial open sets, $\tau 21-\tau 23$ are classes of topologies with three non-trivial open sets where one is a singleton element, $\tau 12, \tau 13$, and $\tau 17$ are classes of topologies with three non-trivial open sets where two are singleton elements, $\tau 9-\tau 11, \tau 14-\tau 16$ and $\tau 18-\tau 20$ are classes of topologies with two non-trivial open sets where one is singleton element. And the other double elements, $\tau 3-\tau 8$ are classes of topologies with one non-trivial open sets, and finally $\tau 2$ is a trivial topology, that is least topology contain in every topological space. 


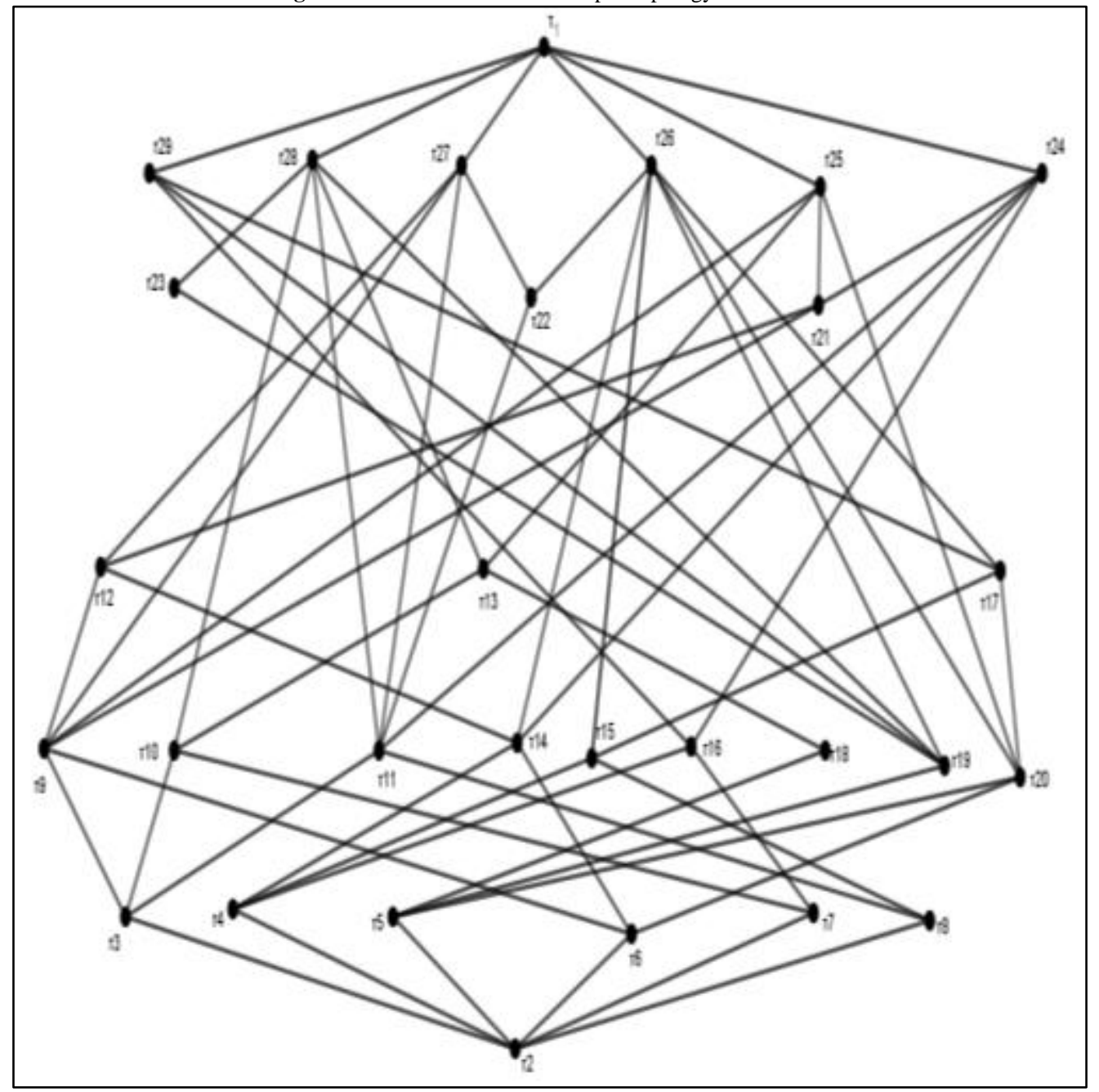

\subsection{Results}

Table-3.11. Number of k-elements on Open topological Space for $T(n, k)$ where $k \geq 1$ and $n \leq 4$

\begin{tabular}{|c|c|c|c|c|c|c|c|c|c|c|c|c|c|c|c|c|c|}
\hline \multicolumn{18}{|c|}{ Number of k-element for $k \geq 1$} \\
\hline & $\begin{array}{c}k \\
=1\end{array}$ & $\begin{array}{l}k \\
=2\end{array}$ & $\begin{array}{l}k \\
=3\end{array}$ & \begin{tabular}{l|}
$k$ \\
$=4$
\end{tabular} & $\begin{array}{l}k \\
=5\end{array}$ & $\begin{array}{l}k \\
=6\end{array}$ & 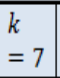 & $\begin{array}{l}k \\
=8\end{array}$ & $\begin{array}{l}k \\
=9\end{array}$ & $\begin{array}{l}k \\
=10\end{array}$ & 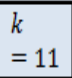 & $\begin{array}{l}k \\
=12\end{array}$ & $\begin{array}{l}k \\
=13\end{array}$ & $\begin{array}{l}\begin{array}{l}k \\
=14\end{array} \\
\end{array}$ & $\begin{array}{l}k \\
=15\end{array}$ & $\begin{array}{l}k \\
=16\end{array}$ & $\mathrm{~T}(\mathrm{n})$ \\
\hline $\begin{array}{l}n \\
=1\end{array}$ & 0 & 1 & & & & & & & & & & & & & & & 1 \\
\hline $\begin{array}{l}n \\
=2\end{array}$ & 0 & 1 & 2 & 1 & & & & & & & & & & & & & 4 \\
\hline $\begin{array}{l}n \\
=3\end{array}$ & 0 & 1 & 6 & 9 & 6 & 6 & 0 & 1 & & & & & & & & & 29 \\
\hline $\begin{array}{l}n \\
=4\end{array}$ & 0 & 1 & 14 & 43 & 60 & 73 & 54 & 54 & 20 & 24 & 0 & 12 & 0 & 0 & 0 & 1 & 335 \\
\hline
\end{tabular}

\section{Number of k-Set in Clopen Set of a Topological Space for $n \leq 4$}

Equation (3) are open by definition. Taking the compliment of equation (3) we have:

$$
\begin{aligned}
\tau_{1}^{c} & =\{X, b, \emptyset\} \\
\tau_{2}^{c} & =\{X, a, \varnothing\} \\
\tau_{3}^{c} & =\{X, b, a, \emptyset\} \\
\tau_{4}^{c} & =\{X, \emptyset\}
\end{aligned}
$$

Equation (16) closed by definition

$$
\begin{aligned}
& \tau_{1}^{c o}=\{X, \emptyset\} \\
& \tau_{2}^{c o}=\{\varnothing, X\} \\
& \tau_{3}^{c o}=\{\varnothing, a, b, X\} \\
& \tau_{4}^{c o}=\{\varnothing, X\}
\end{aligned}
$$


Equation (17) is clopen by definition. It is the intersection of equation (3) and (16) and the topologies formed are represented with Figure 4.1. the set of two element on $\mathrm{X}$ which are clopen has two forms of topologies which are $\tau_{1}, \tau_{2}$ and $\tau_{4}$ known as trivial topological space, they has two elements which are the empty set and the basis set. They has two element each $(k=2)$. The next is $\tau_{3}$ it is known as discrete topology because it contained every element that can be form on X. and it contains four elements $(k=4)$.

Figure-4.1. Schematic nature of clopen topology on three Set

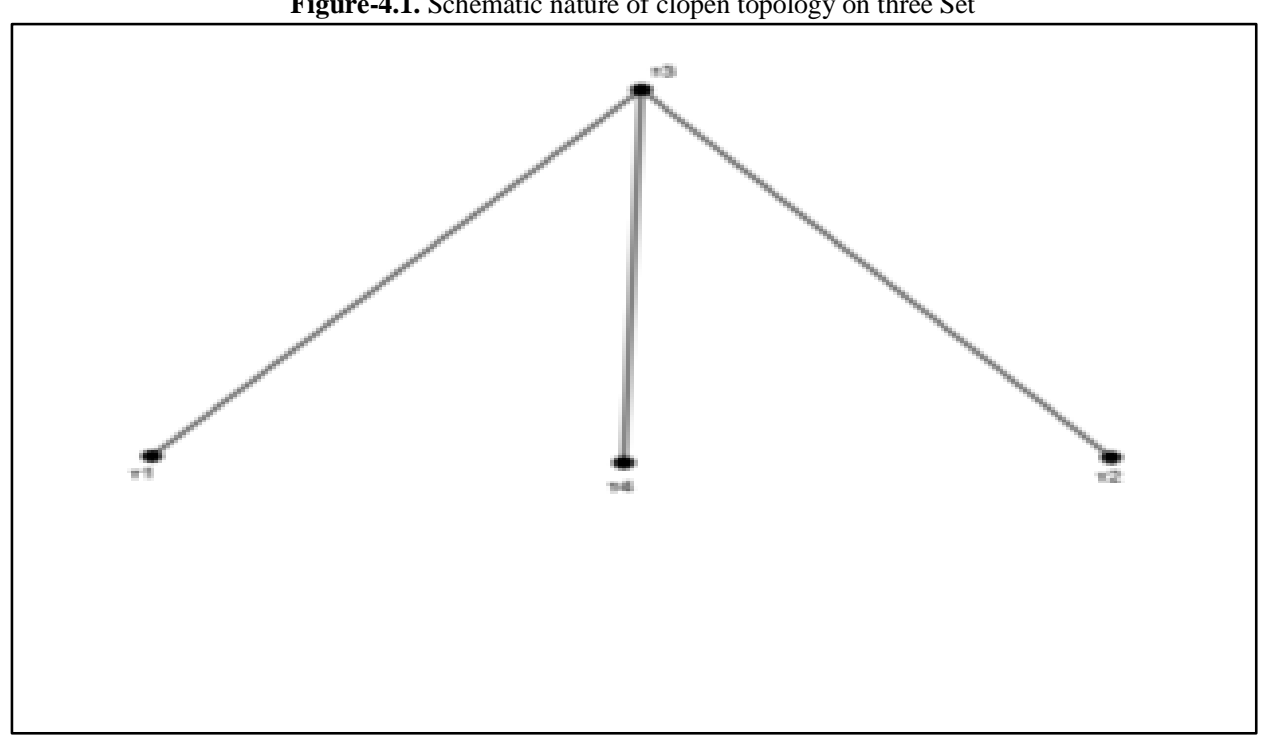

\subsection{Theorem}

Given a set $\mathrm{X}$ with three elements $\{a, b\}$. Such that all topologies formed on $\mathrm{X}$ are clopen on $\mathrm{X}$, and also satisfies the properties of a topological space on X. Then there exist two topological classes on X.

\subsection{Theorem}

Let $(x, \tau)$ be a topological space, then $T(n, k)$ is clopen if and only if $k=2^{n}$ for $n \geq 1$

\subsubsection{Proof}

The clopeness of $T(n, k)$ implies that for any $A \epsilon T(n, k), \quad A^{c} \in T(n, k)$, $A \notin T(n, k)$, if $k \neq 2^{n}$ and $A^{c} \notin T(n, k)$ by contradiction $T(n, k)$ is not clopen if $k \neq 2^{n}$

\subsection{Theorem}

Given a set $\mathrm{X}$ with three elements $\{a, b, c\}$. Such that all topologies formed on $\mathrm{X}$ are clopen on $\mathrm{X}$, and also satisfies the properties of a topological space on X. Then there exist three topologies classes on X.

\subsubsection{Proof}

For a clopen topological space on $n=3$. The graph of the relation between topologies is less cumbersome. Open topologies and closed topologies are made up seven classes. Since the intersection of open and closed gives clopen. Then, that makes it less complicated. Figure 4.4 is a graph of the relation between clopen topological spaces for with three elements. The graph shows how topological spaces of set with three elements are linked to the other. $\tau 1$ is distinct; it contains all the subset formed in every other topology. $\tau 21-\tau 29$ are classes of topologies with two non-trivial open sets where one is singleton element and the other double elements, while. $\tau 2-\tau 20$ are trivial topologies. Since, there are three set of topologies. $\tau 1$ the discrete., nine topologies having four-elements and Nineteen topologies with trivial element. Hence, there are there are three classes of topologies on clopen topologies with three elements. 


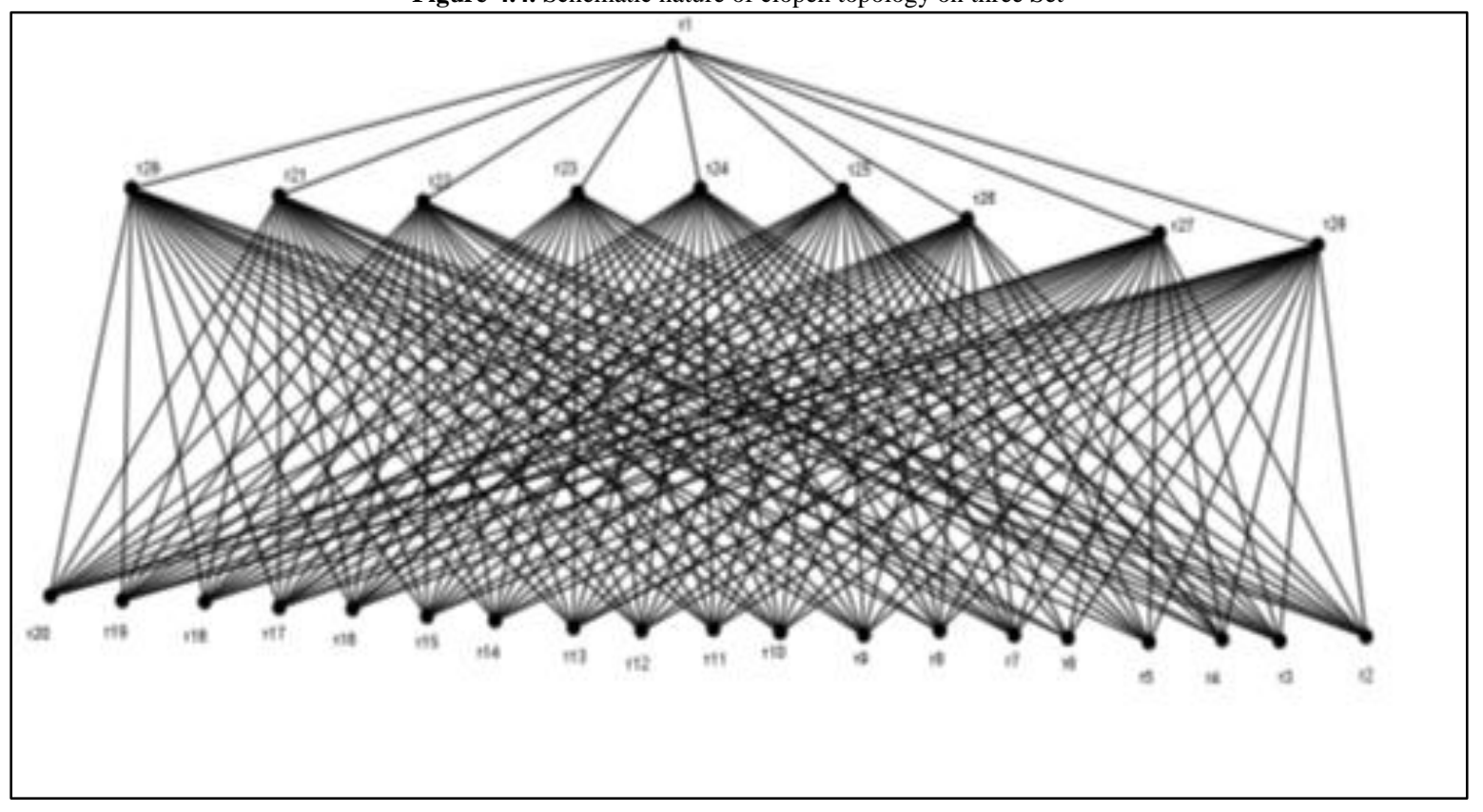

\subsection{Results}

Table-4.5. the analysis shows that values exist on only power set for clopen topologies

\begin{tabular}{|c|c|c|c|c|c|c|c|c|c|c|c|c|c|c|c|c|c|}
\hline \multicolumn{18}{|c|}{ Number of k-element on clopen set for $k \geq 1$} \\
\hline & $\begin{array}{c}k \\
=1\end{array}$ & $\begin{array}{l}\begin{array}{l}k \\
=2\end{array} \\
\end{array}$ & $\begin{array}{l}k \\
=3\end{array}$ & $\begin{array}{l}k \\
=4\end{array}$ & $\begin{array}{l}k \\
=5\end{array}$ & $\begin{array}{l}k \\
=6\end{array}$ & $\begin{array}{l}k \\
=7\end{array}$ & $\begin{array}{l}k \\
=8\end{array}$ & $\begin{array}{l}k \\
=9\end{array}$ & $\begin{array}{l}k \\
=10\end{array}$ & $\begin{array}{l}k \\
=11\end{array}$ & $\begin{array}{l}k \\
=12\end{array}$ & $\begin{array}{l}k \\
=13\end{array}$ & $\begin{array}{l}k \\
=14\end{array}$ & $\begin{array}{l}k \\
=15\end{array}$ & $\begin{array}{l}k \\
=16\end{array}$ & $\mathrm{~T}(\mathrm{n})$ \\
\hline $\begin{array}{l}n \\
=1\end{array}$ & 0 & 1 & & & & & & & & & & & & & & & 1 \\
\hline $\begin{array}{l}n \\
=2\end{array}$ & 0 & 3 & 0 & 1 & & & & & & & & & & & & & 4 \\
\hline $\begin{array}{l}n \\
=3\end{array}$ & 0 & 19 & 0 & 9 & 0 & 0 & 0 & 1 & & & & & & & & & 29 \\
\hline $\begin{array}{l}n \\
=4\end{array}$ & 0 & 219 & 0 & 126 & 0 & 0 & 0 & 9 & 0 & 0 & 0 & 0 & 0 & 0 & 0 & 1 & 335 \\
\hline
\end{tabular}

\section{Conclusion}

In this paper, we take a close look at open and clopen topological spaces. We then study and computed the number of k-set define on open and clopen topological spaces for $n \leq 4$ elements. Our results are basically obtained by hand. Calculations may be refined for $n \geq 4$

\section{References}

[1] Listing, J. B., 1848. "Vorstudien zur Topologies, Vandenhoeck und Reprecht, Go "ttingen." p. 67.

[2] Sharp, H. J., 1968. "Cardinality of finite topologies." Journal of Combinatorial Theory, vol. 5, pp. 82-86.

[3] Kolli, M., 2007. "Direct and elemenatary approach." Computational and Applied Mathematics Journal, vol. 1, pp. $174-179$.

[4] Stanley, P. R., 1971. "On the number of open sets of finite topological spaces." Journal of Combinatorial Theory, vol. 10, pp. 75-79.

[5] Benoumhani, M., 2006. "The number of topologies on finite set." Journal of Interger Sequences, vol. 9, pp.1-9.

[6] Benoumhani, M. and Kolli, M., 2010. "Partitions and topologies." Integer Journal of Integer Sequences, vol. 13, pp.1-19.

[7] Ali-Ahmad, A. F., 2017. "The number of fuzzy clopen sets in fuzzy topological space." Journal of Mathematical Sciences and Applications, vol. 5, pp. 24-26.

[8] Sloane, N. J. A. "Online enclyclopedia of integer sequences." Available: http://www.research.att.com/njas/sequences/index.html 